\title{
Optimal Replenishment Strategy for Inventory Mechanism with a Known Price Increase and Backordering in Finite Horizon
}

\author{
Lixia Zhang $\mathbb{D}^{1}$ and Bo Feng $\mathbb{D}^{2}$ \\ ${ }^{1}$ Department of Mathematics, Jining University, Qufu 273155, Shandong, China \\ ${ }^{2}$ Business and Law Faculty, Deakin University, Locked Bag 20000, Geelong, Australia \\ Correspondence should be addressed to Lixia Zhang; zhlixia66@163.com
}

Received 19 March 2021; Revised 8 November 2021; Accepted 6 December 2021; Published 12 January 2022

Academic Editor: Arkadiusz Zak

Copyright (c) 2022 Lixia Zhang and Bo Feng. This is an open access article distributed under the Creative Commons Attribution License, which permits unrestricted use, distribution, and reproduction in any medium, provided the original work is properly cited.

\begin{abstract}
For the finite horizon inventory mechanism with a known price increase and backordering, based on minimizing the inventory cost, we establish two mixed integer optimization models. By buyer's cost analysis, we present the closed-form solutions to the models, and by comparing the minimum cost of the two strategies, we provide an optimal ordering policy to the buyer. Numerical examples are presented to illustrate the validity of the model, and sensitivity analysis on major parameters is also made to show some insights to the inventory model.
\end{abstract}

\section{Introduction}

Generally, the prices of grain crops such as wheat, corn, and soybeans are low during the first few months after harvest, and the prices will increase thereafter till the next harvest season, and so on. In view of the situation, the buyer should decide the ordering policy so that his inventory cost is as low as possible. This problem can also be stated as that a supplier announces an impending price increase in the future, and the buyer should decide whether to purchase additional stock before the price increase. Further, since wheat, corn, and soybeans are harvested once a year, the buyer's inventory planning time is generally one year. In this paper, we consider the inventory problem with backordering in a finite horizon in which the seller announces the price will increase permanently and the buyer can have a special order before it happens.

The inventory model with known price increase has been frequently discussed in the literature. Possibly, the first work performed in this field was done by Naddor [1] and Brown [2]. They assumed that the price of goods would increase in the future and a buyer would have an opportunity to make a special order forehand. Taylor and Bradley [3] extended the model and obtained the optimal ordering strategies for situations where the price increase does not coincide within the end of an EOQ cycle. Erel [4] investigated the effects of continuous changes of the purchasing price and holding cost on the optimal order quantity and annual cost. Tersine [5] proposed an economic production quantity model under an announced price increase. Shah [6] developed a discretetime stochastic inventory model for perishable items when the vendor announces a price increase at some future time. Huang et al. [7] considered an infinite horizon deterministic inventory model with an announced price increase which assumes that the special order is an integral multiple of the new EOQ quantity. Abad [8] considered a supply chain model in which the producer considers temporary reduction and increase in unit purchasing cost separately and the buyer places a special order in both situations. Chung et al. [9] investigated the buyer's selling policy in response to the future price increase for deteriorating items when the buyer can make a special order before the price increase happens. Ouyang et al. [10] explored the possible effects of price increases on a retailer's replenishment policy when the special order quantity is limited and the rate of deterioration of the goods is assumed to be constant. Wang et al. [11, 12] considered an inventory mechanism with a nondeterministic short-term price discount. 
For the basic EOQ model with backordering of demand during stockout periods, Ghosh [13] presented an infinite horizon deterministic inventory model that handles inventory shortages under an announced price increase. Sharma [14] developed a production inventory model with partial backordering in which the suppliers can increase or temporarily decrease the prices and the buyers can make a special order. A comprehensive survey on this was made by Pentico and Drake [15]. Taleizadeh et al. [16] considered an EOQ problem with backordering under partial delayed payment.

For the finite horizon deterministic inventory model, Lev and Soyster [17] considered the inventory model on determining the optimal ordering policies based on known information about imminent price increase without shortages. Arcelus, Pakkala, and Srinivasan [18] showed the potential differences between the profit-maximizing and the cost-minimizing solutions to the deterministic finite horizon inventory problem, and Arcelus et al. [19] considered the inventory model over a finite horizon under one-time cost changes. Lev and Weiss [20] considered the extensive inventory models with cost changes for a finite horizon and an infinite horizon.

Furthermore, for the basic EOQ model that considers both price changes and backordering, Taleizadeh and Pentico et al. [21] extended classic economic order quantity (EOQ) model with partial backordering in an infinite horizon, in which the supplier announces the price will increase permanently and the retailer can have a special order before it happens. Taleizadeh et al. [22] extended the situation in [21] to probabilistic replenishment intervals further. Taleizadeh et al. [23] considered the rate of demand in the model was assumed proportional to the unit purchasing cost and partial backordering was allowed as a fixed parameter. However, the length of the inventory system operating time is infinite in these articles.

This paper considers the inventory model with backordering in finite horizon, in which a permanent price increase will take place at or before buyer's next scheduled ordering time, and the buyer may have a special order before that. For this set, to maximize the buyer's inventory profit, the buyer should make a tradeoff between enjoying the benefit of the low ordering price and bearing the increase of inventory holding cost lead by the special order. On the basis of the inventory cost analysis, we establish an optimization inventory model based on minimizing the inventory cost and derive a closed-form solution to the model. Some numerical experiments are made to illustrate the validity of the model.

The remainder of the paper is organized as follows. Section 2 presents the assumptions of our model and notations used in the subsequent analysis. Section 3 considers an inventory model with backordering and without price increase in a finite planning horizon and give the optimal solution which will be used in solving the proposed model. In Section 4, we derive a global optimal solution for our concerned inventory models. We derive a solving algorithm and thus provide an optimal replenishment policy to the buyer in Section 5. Numerical experiments on sensitivity analysis are given in Section 6 to show the validity of the models. The conclusions and some extensions are given in the last section.

\section{Notations, Assumptions, and Problem Formulation}

First, we present the notations and assumptions used in this paper (Table 1).

The followings are the assumptions imposed on the concerned inventory model.

Assumption 1. For the inventory system, we assume that

(1) The time horizon is finite;

(2) The leading time for each order is zero;

(3) Shortage and backordered are allowed except for the last replenishment cycle;

(4) The fixed ordering cost and the unit backordering cost for regular order are, respectively, the same as those for special order;

(5) The selling price does not change;

(6) The increase of purchasing price occurs at or before the retailer's next ordering time, which means that there is no opportunity for a regular replenishment before the price increase;

(7) When price increases, it will last to the end of the inventory mechanism;

(8) The stock level is zero at the beginning and end of the inventory system.

Without loss of generality, we assume that the holding cost per item per unit time is proportional with the purchasing cost. Then in the following, we take $h=h_{0}+0.4\left(c-c_{0}\right) / c_{0}$.

From the assumptions on the concerned models, we know that the planning horizon consists of two stages $\left[0, t_{0}\right]$ and $\left(t_{0}, T\right]$, where the ordering price $c_{0}$ in the first stage is strictly less than $c\left(>c_{0}\right)$ in the second stage. When the supplier announces the increase of the purchasing price at $t_{0}$, there are two possible strategies by the buyer: the buyer places a special order under the current lower price before it increases to cut the cost, or the buyer does not place a special order if the remaining stock level at $t_{0}$ is too high or small price increase.

Strategy 1. A special order is not placed at $t_{0}$.

If the buyer does not place a special order at $t_{0}$, he will use a "new" inventory mechanism with backordering in a finite horizon based on the increased price after the current price cycles end.

Strategy 2. A special order is placed at $t_{0}$.

If the buyer adopts this strategy, he will use a "new" inventory mechanism with backordering in a finite horizon based on the increased price after special order cycle ends too. 
TABle 1: Notation.

\begin{tabular}{lc}
\hline Parameters & Descriptions \\
\hline$\lambda$ & Demand rate \\
Fixed order cost & Regular purchasing price \\
$c_{0}$ & Purchasing price after increase \\
$h_{0}$ & Regular holding cost per item per unit time \\
$h$ & Holding cost per item per unit time under increased price \\
$w$ & Backorder cost per unit item per unit time \\
$T$ & Planning horizon of the inventory system \\
$t_{0}$ & The time when purchasing price changes \\
$q_{0}$ & Remaining stock at time $t_{0}$ \\
$Q_{0}$ & Order size before $t_{0}$ \\
$B_{0}$ & Maximum shortage level for a normal order at the current price before $t_{0}$ \\
Decision variables & Special order size \\
$Q_{s}$ & Order size after $t_{0}$ when the special order is not placed \\
$Q$ & Order size after special order \\
$Q^{\prime}$ & Maximum shortage level for a special order at the current price \\
$B_{s}$ & Maximum shortage level for an order at the new price after $t_{0}$ when the special order is not placed \\
$B$ & Maximum shortage level for an order at the new price when a special order is placed \\
$B^{\prime}$ & Inventory cost over interval $\left[t_{0}, T\right]$ without special order \\
Other variables & Invery over interval $\left[t_{0}, T\right]$ with special order \\
$F$ &
\end{tabular}

Since we suppose the unit selling price does not change and the stock level is zero at the beginning and the end of the inventory system, so the method we used is computing the difference of the minimal total costs under two strategies and choosing a strategy with the least cost to save more costs.

In order to make the solution process of solving this problem clearer, we will first provide a solution method for an inventory model with backordering in finite horizon without price increase in Section 3 then exploit these conclusions for giving a solution method to the problem in Section 4.

\section{Solution Method for Inventory Model with Backordering in a Finite Horizon}

In this section, we mainly consider the inventory mechanism with backordering in a finite horizon of length $T$ and without purchasing price variation. Also, for the need discussed in the next section, we assume that the initial stock is $q$, and the stock is zero at the end of the inventory mechanism. See Figures 1 and 2 .

For this setting, suppose that $k$ orders are placed in the $\left[t_{0}, T\right]$ with order sizes $Q_{1}, Q_{2}, \cdots, Q_{k}$, and the maximum shortage levels at replenishment cycles are $B_{1}, B_{2}, \cdots, B_{k}$, respectively. Since the demand rate is invariant, shortage is fully backordered, and the stock is zero at the end of the inventory mechanism (i.e. $\left.B_{k}=0\right)$, so

$$
Q_{1}+Q_{2}+\cdots+Q_{k}=\lambda T-q .
$$

From the knowledge of inventory management [24], we can obtain the total operating cost over the planning horizon $T$

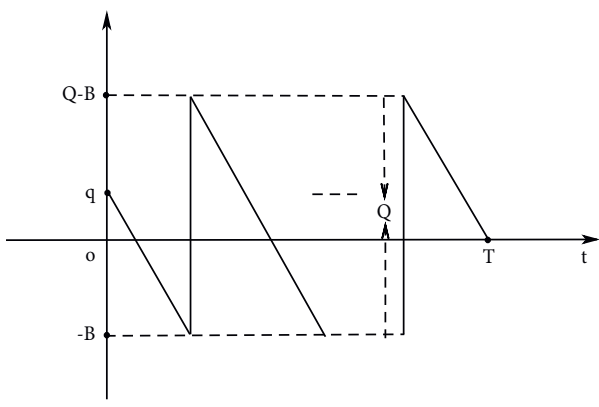

FIGURE 1: Ordering policy for $q \geq 0$.

$$
\begin{aligned}
& F\left(k, Q_{1}, \ldots, Q_{k}, B_{1}, \ldots, B_{k-1}\right) \\
& =k A+c \sum_{i=1}^{k} Q_{i}+\frac{h}{2 \lambda}\left(Q_{1}+q\right)^{2} \\
& +\frac{h}{2 \lambda} \sum_{i=2}^{k}\left(Q_{i}-B_{i-1}\right)^{2}+\frac{w}{2 \lambda} \sum_{i=1}^{k-1} B_{i}^{2} \\
& =c(\lambda T-q)+k A+\frac{h}{2 \lambda}\left(Q_{1}+q\right)^{2} \\
& ++\frac{h}{2 \lambda} \sum_{i=2}^{k}\left(Q_{i}-B_{i-1}\right)^{2}+\frac{w}{2 \lambda} \sum_{i=1}^{k-1} B_{i}^{2} .
\end{aligned}
$$

To minimize the buyer's operating cost, we only need to determine the optimal order times $k$, the sizes $Q_{i}(i=1, \ldots, k)$, and the maximum shortage levels $B_{i}(i=1, \ldots, k-1)$, which can be formulated as the following optimization problem: 


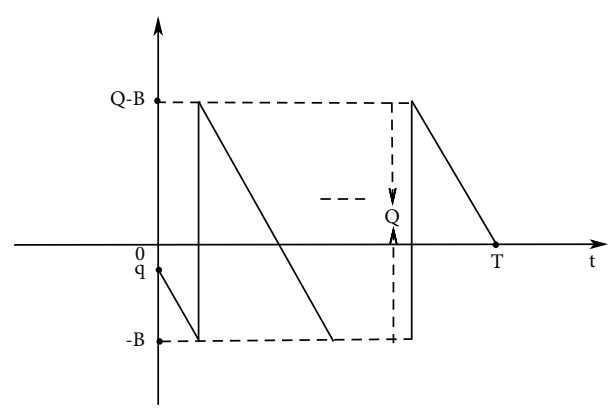

Figure 2: Ordering policy for $q<0$.

$$
\begin{aligned}
& \min _{k, Q_{i}, B_{i}} F\left(k, Q_{1}, \ldots, Q_{k}, B_{1}, \ldots, B_{k-1}\right) \\
& \text { s.t. } \quad Q_{1}+Q_{2}+\cdots+Q_{k}=\lambda T-q, \\
& Q_{i} \geq 0, i=1, \ldots, k, \\
& B_{i} \geq 0, i=1, \ldots, k-1, \\
& \quad k \text { is a positive integer. }
\end{aligned}
$$

This is a nonlinear mixed integer optimization problem. For this problem, we have the following conclusion.

Theorem 1. For an inventory model with backordering in finite horizon, i.e., inventory model (3), all the optimal maximum shortage levels are the same except the last one, and all the optimal ordering sizes are the same except the first one, i.e.,

$$
\begin{aligned}
& Q_{2}=\cdots=Q_{k} \triangleq Q, \\
& B_{1}=\cdots=B_{k-1} \triangleq B, \\
& Q_{1}=Q-B-q .
\end{aligned}
$$

Furthermore, the optimal ordering size, maximum shortage level, and the optimal order time are, respectively,

$$
\begin{aligned}
& Q=\frac{(w+h) \lambda T}{k w+(k-1) h}, \\
& B=\frac{h \lambda T}{k w+(k-1) h}, \\
& k=\left\lfloor T \sqrt{\frac{w \lambda h}{2 A(w+h)}}+\frac{h}{w+h}\right\rfloor \\
& \quad \text { or }\left\lceil T \sqrt{\frac{w \lambda h}{2 A(w+h)}}+\frac{h}{w+h}\right\rceil,
\end{aligned}
$$

and the minimum inventory cost within the horizon $[0, T]$ is

$$
F=c(\lambda T-q)+k A+\frac{w h \lambda T^{2}}{2[k w+(k-1) h]} .
$$

Proof. For completeness, we include the proof of the lemma in Appendix A.

We will exploit the conclusion of Theorem 1 repeatedly in the remainder of this paper. For convenience, the optimal ordering policy given in Theorem 1 for the inventory mechanism with backordering in the finite horizon and without purchasing price variation is denoted by "BEOQ" in the subsequent sections.

\section{Solution Method for Inventory Model with a Known Price Increase and Backordering in Finite Horizon}

For this inventory system, there are two ordering strategies according to whether a special order is placed or not at $t_{0}$, as shown in Figures 3 and 4 . If the buyer places a special order at $t_{0}$, the special order cycle will end at $t_{2}$, and the inventory cycles based on the current lower price end at $t_{1}$ if the buyer does not place a special order. It is easy to calculate that $t_{1}=t_{0}+q_{0}+B_{0} / \lambda, \quad t_{2}=t_{0}+q_{0}+Q_{s}+B_{s} / \lambda$. For convenience, we denote $T_{0}=T-t_{0}, T_{1}=T-t_{1}$, and $T_{2}=T-t_{2}$.

Since shortages are allowed, two possible scenarios may occur at $t_{0}$ : the price increase for the item occurs when there is still inventory or there is a shortage, that is:

Scenario 1: $q_{0} \geq 0$, and Scenario 2: $q_{0}<0$.

In the subsequent analysis, we will first discuss the minimum inventory costs of the two strategies, then compare the difference in the two minimum cost, and finally, choose the optimal ordering strategy.

4.1. Strategy 1: A Special Order Is Not Placed at $t_{0}$. For this strategy, when the inventory cycles under the current price end at $t_{1}$, the buyer will continue using a new BEOQ order policy till the end of the inventory system, so that the inventory cost over the interval $\left[t_{0}, T\right]$ consists of inventory cost over two intervals $\left[t_{0}, t_{1}\right)$ and $\left[t_{1}, T\right]$.

Scenario 1. $q_{0} \geq 0$

Under this scenario, the price increases when there is no shortage, and the remaining stock $q_{0}$ at $t_{0}$ will be postponed to the second stage. By the knowledge of inventory control, we can calculate that the inventory cost during $\left[t_{0}, t_{1}\right)$ is

$$
f_{1}=\frac{h_{0}}{2 \lambda} q_{0}^{2}+\frac{w}{2 \lambda} B_{0}^{2} .
$$

Since the stock level is $-B_{0}$ at $t_{1}$, the inventory system will run a new "BEOQ" order policy during the horizon $\left[t_{1}, T\right]$ under the new price $c$, and the stock level is $-B_{0}$ and 


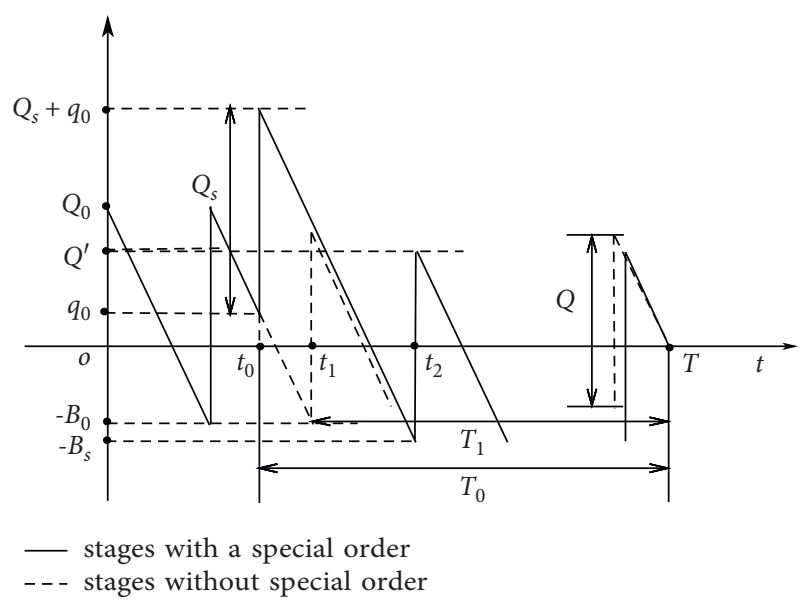

FIgURE 3: Ordering policy for $q_{0} \geq 0$.

zero at the beginning and end of the planning horizon, respectively; see Figures 3 and 4 .

For this scenario, when the special order is not placed at $t_{0}$, we denote the inventory cost during this period from $t_{0}$ to the end by $F$, which is a function of order quantity $Q_{1}, \ldots, Q_{n}$ and maximum shortage level $B_{1}, \ldots, B_{n-1}$ for an order after $t_{0}$. Using an approach similar to that used and according to formula (2) in Section 3, then

$$
\begin{aligned}
& g\left(n, Q_{1}, \ldots, Q_{n}, B_{1}, \ldots, B_{n-1}\right) \\
& =n A+c \sum_{i=1}^{n} Q_{i}+\frac{h}{2 \lambda}\left(Q_{1}-B_{0}\right)^{2} \\
& +\frac{h}{2 \lambda} \sum_{i=2}^{n}\left(Q_{i}-B_{i-1}\right)^{2}+\frac{w}{2 \lambda} \sum_{i=1}^{n-1} B_{i}^{2} \\
& =c\left(\lambda T+B_{0}\right)+n A+\frac{h}{2 \lambda}\left(Q_{1}-B_{0}\right)^{2} \\
& \quad+\frac{h}{2 \lambda} \sum_{i=2}^{n}\left(Q_{i}-B_{i-1}\right)^{2}+\frac{w}{2 \lambda} \sum_{i=1}^{n-1} B_{i}^{2} .
\end{aligned}
$$

So the ordering policy during the horizon $\left[t_{1}, T\right]$ of the concerned model can be formulated as the following optimization model:

$$
\begin{aligned}
& \min _{n, Q_{i}, B_{i}} g\left(n, Q_{1}, \ldots, Q_{n}, B_{1}, \ldots, B_{n-1}\right), \\
& \text { s.t. } \quad Q_{1}+Q_{2}+\cdots+Q_{n}=\lambda T_{1}+B_{0}, \\
& Q_{i} \geq 0, i=1, \ldots, n, \\
& B_{i} \geq 0, i=1, \ldots, n-1, \\
& n \text { is a positive integer. }
\end{aligned}
$$

According to formulas (5) and (6) of Theorem 1 in Section 3, we know that all the optimal ordering sizes are the same except the first one, all the optimal maximum shortage

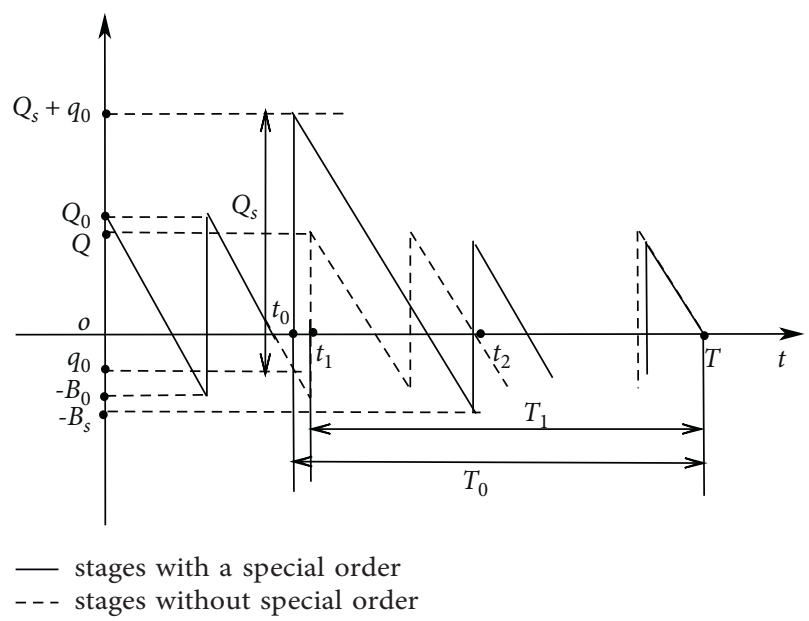

FIgURE 4: Ordering policy for $q_{0}<0$.

levels are the same, and the optimal ordering size, maximum shortage level, and the optimal order time over the interval $\left[t_{1}, T\right]$, respectively, are

$$
\begin{aligned}
Q= & \frac{(w+h) \lambda T_{1}}{n w+(n-1) h}, \\
B= & \frac{h \lambda T_{1}}{n w+(n-1) h}, \\
n= & \left\lfloor T_{1} \sqrt{\frac{w \lambda h}{2 A(w+h)}}+\frac{h}{w+h}\right\rfloor \triangleq n_{1} \quad \text { or } \\
& \left\lceil T_{1} \sqrt{\frac{w \lambda h}{2 A(w+h)}}+\frac{h}{w+h}\right\rceil \triangleq n_{2},
\end{aligned}
$$

and the minimum inventory cost during the interval $\left[t_{1}, T\right)$ is

$$
g_{1}=c\left(\lambda T_{1}+B_{0}\right)+n_{1} A+\frac{w h \lambda T_{1}^{2}}{2\left[n_{1} w+\left(n_{1}-1\right) h\right]},
$$

or

$$
g_{2}=c\left(\lambda T_{1}+B_{0}\right)+n_{2} A+\frac{w h \lambda T_{1}^{2}}{2\left[n_{2} w+\left(n_{2}-1\right) h\right]} .
$$

If $g_{1} \leq g_{2}$, then the optimal ordering times and the minimum inventory cost during the interval $\left[t_{1}, T\right)$ are $n^{*}=$ $n_{1}$ and $g_{1}$, respectively; otherwise, they are $n^{*}=n_{2}$ and $g_{2}$. Denote the minimum inventory cost during horizon $\left[t_{1}, T\right)$ by

$$
g^{*}=c\left(\lambda T_{1}+B_{0}\right)+n^{*} A+\frac{w h \lambda T_{1}^{2}}{2\left[n^{*} w+\left(n^{*}-1\right) h\right]} .
$$

In summary, under strategy 1 , if the remaining inventory stock at $t_{0}$ is nonnegative, the minimum inventory cost over the horizon $\left[t_{0}, T\right]$ is 


$$
\begin{aligned}
F^{*}= & f_{1}+g^{*} \\
= & c\left(\lambda T_{1}+B_{0}\right)+n^{*} A+\frac{h_{0}}{2 \lambda} q_{0}^{2} \\
& +\frac{w}{2 \lambda} B_{0}^{2}+\frac{w h \lambda T_{1}^{2}}{2\left[n^{*} w+\left(n^{*}-1\right) h\right]} .
\end{aligned}
$$

Scenario 2. $q_{0}<0$.

Under this scenario, the stock position at $t_{0}$ is exhausted; therefore the inventory cost during $\left[t_{0}, t_{1}\right)$ needs to subtract holding costs (see Figure 4), so that the inventory cost during $\left[t_{0}, t_{1}\right)$ is

$$
f_{2}=\frac{w}{2 \lambda} B_{0}^{2}-\frac{h_{0}}{2 \lambda} q_{0}^{2}
$$

Yet, if there is a shortage at $t_{0}$, again using an approach similar to that used in Section 3, the minimum inventory cost during the interval $\left[t_{1}, T\right)$ can still be expressed as $g^{*}$; thus the minimum inventory cost over the horizon $\left[t_{0}, T\right]$ is

$$
\begin{aligned}
F^{*}= & f_{2}+g^{*} \\
= & c\left(\lambda T_{1}+B_{0}\right)+n^{*} A-\frac{h_{0}}{2 \lambda} q_{0}^{2} \\
& +\frac{w}{2 \lambda} B_{0}^{2}+\frac{w h \lambda T_{1}^{2}}{2\left[n^{*} w+\left(n^{*}-1\right) h\right]} .
\end{aligned}
$$

Obviously, both $f_{1}$ and $f_{2}$ are constants and independent of decision variables; therefore, minimizing inventory cost during the period $\left[t_{0}, T\right]$ is equivalent to minimizing the buyer's inventory cost over the interval $\left[t_{1}, T\right]$.

Based on the discussion above, we can obtain the following two candidate optimal ordering policies in the horizon $\left[t_{1}, T\right]$ under strategy 1 .

Policy $\pi_{1}: n^{*}=n_{1}, \quad Q_{1}^{*}=w \lambda T_{1} /\left(n_{1} w+\left(n_{1}-1\right) h\right)+B_{0}$, $Q^{*}=(w+h) \lambda T_{1} /\left(n_{1} w+\left(n_{1}-1\right) h\right)$,

$B^{*}=h \lambda T_{1} /\left(n_{1} w+\left(n_{1}-1\right) h\right)$,

which applies to the case that $g_{1} \leq g_{2}$.
For this order policy, the minimum inventory cost in horizon $\left[t_{1}, T\right]$ is $g^{*}=g_{1}$.

Policy $\pi_{2}: n^{*}=n_{1}, \quad Q_{1}^{*}=w \lambda T_{1} /\left(n_{1} w+\left(n_{1}-1\right) h\right)+B_{0}$, $Q^{*}=(w+h) \lambda T_{1} /\left(n_{1} w+\left(n_{1}-1\right) h\right)$,

$B^{*}=h \lambda T_{1} /\left(n_{1} w+\left(n_{1}-1\right) h\right)$,

which applies to the case that $g_{1}<g_{2}$.

For this order policy, $g^{*}=g_{2}$.

4.2. Strategy 2: A Special Order Is Placed at $t_{0}$. By the inventory management theory, if we place a one-time special order at the current price before the price increases for both scenarios of $q_{0} \geq 0$ and $q_{0}<0$, the inventory cost in the special replenishment cycle can be expressed as

$$
f_{s}=c_{0} Q_{s}+A+\frac{h_{0}}{2 \lambda}\left(Q_{s}+q_{0}\right)^{2}+\frac{w}{2 \lambda} B_{s}^{2} .
$$

Since the special order is exhausted at $t_{2}$ (see Figures 3 and 4 ), the next order will be placed at $t_{2}$; hence, the inventory system will run a new BEOQ inventory mechanism during the period $\left[t_{2}, T\right]$, and the shortage is $B_{s}$ at $t_{2}$.

Suppose that the order sizes $Q_{1}^{\prime}, \ldots, Q_{m}^{\prime}$ and the maximal shortage levels $B_{1}^{\prime}, \ldots, B_{m-1}^{\prime}$ are made after the special order; again using a similar discussion in Section 3, we can obtain the inventory cost in horizon $\left[t_{2}, T\right]$

$$
\begin{aligned}
& g_{s}\left(m, Q_{1}^{\prime}, \ldots, Q_{m}^{\prime}, B_{1}^{\prime}, \ldots, B_{m-1}^{\prime}\right) \\
& =c\left(\lambda T_{2}+B_{s}\right)+m A+\frac{h}{2 \lambda}\left(Q_{1}^{\prime}-B_{s}\right)^{2} \\
& +\frac{h}{2 \lambda} \sum_{i=2}^{m}\left(Q_{i}^{\prime}-B_{i-1}^{\prime}\right)^{2}+\frac{w}{2 \lambda} \sum_{i=1}^{m-1} B_{i}^{\prime 2} \\
& =c\left(\lambda T_{0}-q_{0}-Q_{s}\right)+m A+\frac{h}{2 \lambda}\left(Q_{1}^{\prime}-B_{s}\right)^{2} \\
& +\frac{h}{2 \lambda} \sum_{i=2}^{m}\left(Q_{i}^{\prime}-B_{i-1}^{\prime}\right)^{2}+\frac{w}{2 \lambda} \sum_{i=1}^{m-1} B_{i}^{\prime 2} .
\end{aligned}
$$

Accordingly, the total inventory cost during the period $\left[t_{0}, T\right]$ is

$$
\begin{aligned}
F_{s}\left(m, Q_{s}, B_{s}, Q_{1}^{\prime}, \ldots, Q_{m}^{\prime}, B_{1}^{\prime}, \ldots, B_{m-1}^{\prime}\right)= & f_{s}+g_{s}\left(m, Q_{1}^{\prime}, \ldots, Q_{m}^{\prime}, B_{1}^{\prime}, \ldots, B_{m-1}^{\prime}\right) \\
= & c \lambda T_{0}-c q_{0}-\left(c-c_{0}\right) Q_{s}+(m+1) A+\frac{h_{0}}{2 \lambda}\left(Q_{s}+q_{0}\right)^{2}+\frac{w}{2 \lambda} B_{s}^{2}+\frac{h}{2 \lambda}\left(Q_{1}^{\prime}-B_{s}\right)^{2} \\
& +\frac{h}{2 \lambda} \sum_{i=2}^{m}\left(Q_{i}^{\prime}-B_{i-1}^{\prime}\right)^{2}+\frac{w}{2 \lambda} \sum_{i=1}^{m-1} B_{i}^{\prime 2} .
\end{aligned}
$$

Thus, the problem of determining an optimal ordering policy of strategy 2 can be formulated as the following optimization problem: 


$$
\begin{aligned}
& \min _{m, Q_{s}, B_{s}, Q_{p}^{\prime} B_{i}^{\prime}} F_{s}\left(m, Q_{s}, B_{s}, Q_{1}^{\prime}, \ldots, Q_{m}^{\prime}, B_{1}^{\prime}, \ldots, B_{m-1}^{\prime}\right) \\
& \text { s.t. } Q_{s}+Q_{1}^{\prime}+Q_{2}^{\prime}+\cdots+Q_{m}^{\prime}=\lambda T_{0}-q_{0} \\
& Q_{s} \geq 0, B_{s} \geq 0 \\
& Q_{i}^{\prime} \geq 0, i=1, \ldots, m, \\
& B_{i}^{\prime} \geq 0, i=1, \ldots, m-1, \\
& m \text { is a positive integer. }
\end{aligned}
$$

For problem (21), we have the following conclusion.

Theorem 2. For inventory model (21) with $q_{0} \geq 0$ or $q_{0}<0$, assume $m$ is fixed; after the special order, all optimal maximum shortage levels are the same, and the optimal ordering sizes are the same. Furthermore, the optimal special ordering size is

$$
Q_{s}= \begin{cases}\lambda T_{0}-q_{0}-\frac{m \lambda(w+h) \alpha}{m h_{0}(w+h)+w h}, & \text { if } \alpha>0 \text { and } \beta \leq 0, \\ \lambda T_{0}-q_{0}, & \text { or } \alpha>0, \beta>0 \text { and } m<\delta, \\ & \text { otherwise, }\end{cases}
$$

the ordering size after special order is

$$
Q^{\prime}= \begin{cases}\lambda T_{0}-q_{0}-\frac{\lambda(w+h) \alpha}{m h_{0}(w+h)+w h}, & \text { if } \alpha>0 \text { and } \beta \leq 0, \\ 0, & \text { or } \alpha>0, \beta>0 \text { and } m<\delta, \\ & \text { otherwise, }\end{cases}
$$

and the maximal shortage level is

$$
B_{s}=B^{\prime}= \begin{cases}\frac{\lambda h \alpha}{m h_{0}(w+h)+w h}, & \text { if } \alpha>0 \text { and } \beta \leq 0, \\ 0, & \text { or } \alpha>0, \beta>0 \text { and } m<\delta, \\ & \text { otherwise, }\end{cases}
$$

where $\quad \alpha=h_{0} T_{0}+c_{0}-c, \beta=q_{0} h_{0}+\lambda\left(c_{0}-c\right), \delta=\left(\lambda T_{0}\right.$ $\left.-q_{0}\right) w h /(w+h) \beta$.

Proof. See Appendix B.

From the proof of Theorem 2, if $\alpha \geq 0$ and $\beta \leq 0$, or $\alpha \geq 0, \beta>0$ and $m<\delta$ hold, then

$$
\begin{aligned}
F_{s}= & c \lambda T_{0}+c q_{0}+\left(c_{0}-c\right) Q_{s}+(m+1) A+\frac{h_{0}}{2 \lambda}\left(Q_{s}+q_{0}\right)^{2} \\
& +\frac{m h}{2 \lambda}\left(Q^{\prime}-B^{\prime}\right)^{2}+\frac{m w}{2 \lambda} B^{\prime 2} \\
= & c_{0} \lambda T_{0}-c_{0} q_{0}+(m+1) A+\frac{h_{0} \lambda T_{0}^{2}}{2}-\frac{m \lambda(w+h) \alpha^{2}}{2\left[m h_{0}(w+h)+w h\right]} .
\end{aligned}
$$

Otherwise,

$$
\begin{aligned}
& m=0, Q_{s}=\lambda T_{0}-q, Q^{\prime}=0, B^{\prime}=0, \\
& F_{s}=c_{0} \lambda T_{0}-c_{0} q_{0}+A+\frac{h_{0} \lambda T_{0}^{2}}{2} .
\end{aligned}
$$

Now, we consider the optimal ordering time under condition (58) or (59).

For the function $F_{s}$ given by (25),

$$
F_{s}^{\prime \prime}(m)=\frac{w^{2} h \lambda(w+h) \alpha^{2}}{\left[m h_{0}(w+h)+w h\right]^{3}}>0,
$$

so that $F_{s}(m)$ is a convex function in $m$, and its minimum can be obtained at the stationary point of the function $F_{s}$, which yields

$$
m=\frac{1}{h_{0}} \sqrt{\frac{w \lambda h}{2 A(w+h)}}\left(\alpha-\sqrt{\frac{2 A w h}{\lambda(w+h)}}\right) .
$$

Denote $\gamma=\sqrt{(2 A w h / \lambda(w+h))}$

and $m_{1}=\lfloor m\rfloor, m_{2}=\lceil m\rceil$. Taking the fact $m$ is a positive integer into consideration, we know that the optimal ordering time is $m=m_{1}$ when $F_{s}\left(m_{1}\right) \geq F_{s}\left(m_{2}\right)$; otherwise it is $m=m_{2}$.

Based on the above discussion, and if $\alpha \leq \gamma$, the optimal ordering time is $m^{*}=0$. We obtain the following three candidate optimal order policies in horizon $\left[t_{0}, T\right]$ under strategy 2.

Policy $\pi_{3}$ :

$$
m^{*}=m_{1}, \quad Q_{s}^{*}=\lambda T_{0}-q_{0}-\frac{m_{1} \lambda(w+h) \alpha}{m_{1} h_{0}(w+h)+w h},
$$

$Q^{\prime} *=\frac{\lambda(w+h) \alpha}{m_{1} h_{0}(w+h)+w h}, B^{\prime *}=\frac{\lambda h \alpha}{m_{1} h_{0}(w+h)+w h}$.

It applies to

$$
\begin{gathered}
\alpha>\gamma, \beta \leq 0, \\
F_{s}\left(m_{1}\right) \leq F_{s}\left(m_{2}\right), \\
\text { or } \alpha>\gamma, \beta>0, m_{2}<\delta \text { and } F_{s}\left(m_{1}\right) \leq F_{s}\left(m_{2}\right), \\
\text { or } \alpha>\gamma, \beta>0, m_{1}<\delta \leq m_{2} .
\end{gathered}
$$

For policy $\pi_{3}$, the minimum inventory cost over the interval $\left[t_{0}, T\right]$ is $F_{s}^{*}=F_{s}\left(m_{1}\right)$.

Policy $\pi_{4}$ :

$$
\begin{aligned}
m^{*} & =m_{2}, Q_{s}^{*}=\lambda T_{0}-q_{0}-\frac{m_{2} \lambda(w+h) \alpha}{m_{2} h_{0}(w+h)+w h} \\
Q^{\prime *} & =\frac{\lambda(w+h) \alpha}{m_{2} h_{0}(w+h)+w h}, B^{\prime *}=\frac{\lambda h \alpha}{m_{2} h_{0}(w+h)+w h},
\end{aligned}
$$

which applies to

$$
\alpha>\gamma, \beta \leq 0 \text {, and } F_{s}\left(m_{1}\right)>F_{s}\left(m_{2}\right) \text {, }
$$




$$
\text { or } \alpha>\gamma, \beta>0, m_{2}<\delta \text { and } F_{s}\left(m_{1}\right)>F_{s}\left(m_{2}\right) .
$$

For this policy, the minimum inventory cost over the interval $\left[t_{0}, T\right]$ is $F_{s}^{*}=F_{s}\left(m_{2}\right)$.

Policy $\pi_{5}$ :

$$
m^{*}=0, Q_{s}^{*}=\lambda T_{1}-q_{0}, Q^{\prime *}=B^{\prime *}=0,
$$

i.e., no order is made after the special order; the minimum inventory cost over the interval horizon $\left[t_{0}, T\right]$ is

$$
F_{s}^{*}=c_{0} \lambda T_{0}-c_{0} q_{0}+A+\frac{h_{0} \lambda T_{0}^{2}}{2} .
$$

According to the analysis above, the difference of the minimal total costs under two strategies is $G^{*}=F^{*}-F_{s}^{*}$. Certainly, placing a special order or not is determined by the sign of $G^{*}$. If $G^{*} \geq 0$, then the buyer placing a special order can save costs than not placing special orders, and the candidate optimal policy is $\pi_{3}, \pi_{4}$, or $\pi_{5}$ after the special order.

On the contrary, if $G^{*}<0$, the buyer will not place a special order, and the candidate optimal policy is $\pi_{1}$ or $\pi_{2}$ after the regular order replenishment cycle.

Considering all the ordering policies discussed above, we can present our algorithm for our problem in this paper.

\section{Algorithm}

The algorithm flowchart for problem is shown in Figure 5. See Appendix C.

To compute the remaining stock $q_{0}$ at $t_{0}$, we first give the optimal order policy under the current price. For an inventory mechanism, if there is not price change, this is an inventory model with backordering in the finite horizon of length $T$ under the current price $c_{0}$, and the stock level is zero at the beginning and the end of the inventory system; based on the conclusions (5) and (6) in Theorem 1, the optimal ordering size $Q_{0}$, maximum shortage level $B_{0}$, and the optimal order times $k$ under the current price are, respectively,

$$
\begin{aligned}
Q_{0} & =\frac{\left(w+h_{0}\right) \lambda T}{k w+(k-1) h_{0}}, \\
B_{0} & =\frac{h_{0} \lambda T}{k w+(k-1) h_{0}}, \\
k & =\left\lfloor T \sqrt{\frac{w \lambda h_{0}}{2 A\left(w+h_{0}\right)}}+\frac{h}{w+h_{0}}\right\rfloor \triangleq k_{1} \\
\quad & \text { or }\left\lceil T \sqrt{\frac{w \lambda h_{0}}{2 A\left(w+h_{0}\right)}}+\frac{h_{0}}{w+h_{0}}\right\rceil \triangleq k_{2} .
\end{aligned}
$$

According to these, we can compute that

$$
q_{0}=\left\lceil\frac{\lambda t_{0}}{Q_{0}}\right\rceil Q_{0}-B_{0}-\lambda t_{0} .
$$

\section{Computational Experiments and Sensitivity Analysis}

In this section, in order to show the applicability of the presented models, we will perform some numerical examples and sensitivity analysis on major parameters. Based on the algorithm above, if the basic parameters of the models are given, we can obtain an optimal ordering policy. The results of computational experiments and sensitivity analysis can be obtained by the Matlab procedures of algorithm above, so we mainly list the important results and list the simple solution process.

\subsection{Computational Experiments}

Example 1. Consider the inventory system with the following parameters: $\quad \lambda=500, A=50, c_{0}=10, c=10.5, h_{0}=4$, $h=4.2, w=3, T=12, t_{0}=2.04$.

Solution: For this inventory system, first, we calculate the optimal regular order times, order size, the maximum shortage level before $t_{0}$, and the remaining stock at $t_{0}$ before the price increase does not take place according to formulas (41)-(43) as follows:

$$
k=36, Q_{0}=169.35, B_{0}=96.77, q_{0}=68.71 .
$$

If we adopt strategy 1 , then the candidate optimal policy set is $\left\{\pi_{1}, \pi_{2}\right\}$.

However, if we adopt strategy 2, since $\alpha=39.34>\gamma=0.5916, \quad$ and $\quad \beta=24.84>0, m_{2}=29$ $<\left(\lambda T_{0}-q_{0}\right) w h /(w+h) \beta=346.02$; based on Theorem 2 in Section 4 , the candidate optimal policy set is $\left\{\pi_{3}, \pi_{4}\right\}$. The numerical results by algorithm are listed in Table 2 .

From Table 2, we can see that $F^{*}=54493, F_{s}^{*}=54525$, and thus $G^{*}=-32$; hence the optimal ordering policy of Example 1 is $\pi_{1}$, and the optimal order times, optimal ordering sizes, and the optimal maximum shortage level over the interval $[\mathrm{t} 1, \mathrm{~T}]$ are, respectively,

$$
m^{*}=29, Q_{1}^{*}=167.37, Q^{*}=169.43, B^{*}=98.83 \text {. }
$$

In this way, the buyer's inventory cost savings are 32.

Example 2. For the inventory system considered in Example 1 , set $t_{0}=2.5$ and other parameters remain unchanged.

For this inventory system, the optimal regular order times, order size, and the maximum shortage level are the same as in Example 1, and the remaining stock at $t_{0}$ is $q_{0}=8.06$.

Similar to Example 1, since $\alpha=39.34>\gamma=0.5916$, and $\beta=-375.16<0$, if we apply strategy 2 , the candidate optimal policy set is $\left\{\pi_{3}, \pi_{4}\right\}$. The numerical results are listed in Table 3, from which we can see that the cost of applying $\pi_{3}$ is minimal, so the optimal ordering policy of Example 2 is $\pi_{3}$, and the buyer's inventory cost saving of placing a special order compared to not placing special order is 16 . 


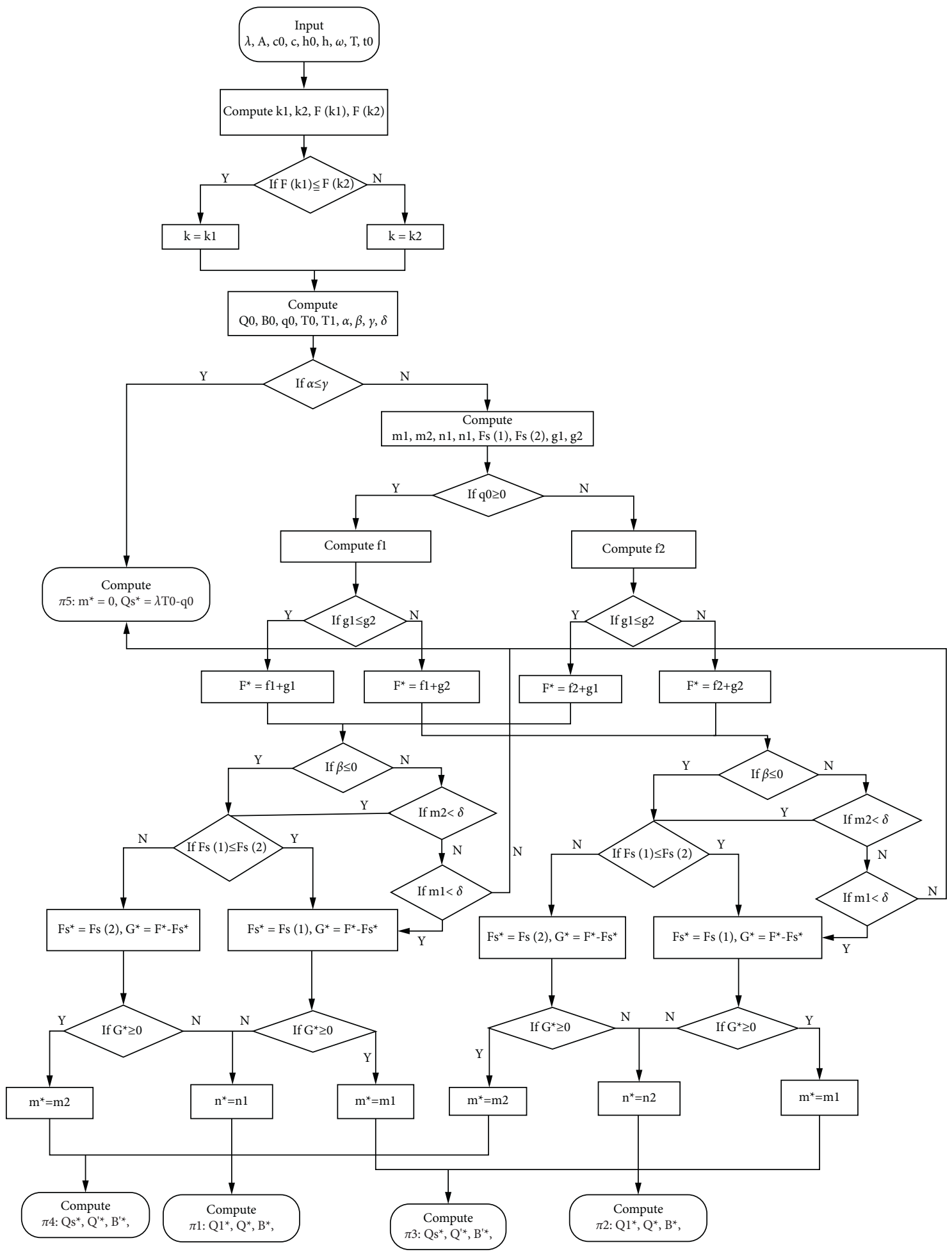

Figure 5: Algorithm flowchart.

TABle 2: Numerical results on Example 1.

\begin{tabular}{lccccc}
\hline Policy & $Q_{s}$ & $m \backslash n$ & $Q \backslash Q^{\prime}$ & $B \backslash B^{\prime}$ & $F \backslash F_{s}$ \\
\hline$\pi_{1}$ & - & 29 & 30 & 169.43 & 98.83 \\
$\pi_{2}$ & - & 28 & 163.67 & 95.47 & 54493 \\
$\pi_{3}$ & 69.44 & 29 & 172.92 & 100.87 & 54494 \\
$\pi_{4}$ & 66.87 & 167.05 & 54.45 & 54525 \\
\hline
\end{tabular}


TABle 3: Numerical result on Example 2.

\begin{tabular}{lccccc}
\hline Policy & $Q_{s}$ & $m \backslash n$ & $Q \backslash Q^{\prime}$ & $B \backslash B^{\prime}$ & $F \backslash F_{s}$ \\
\hline$\pi_{1}$ & - & 28 & 169.43 & 98.83 & 52596 \\
$\pi_{2}$ & - & 29 & 163.67 & 95.47 & 52597 \\
$\pi_{3}$ & 129.18 & 27 & 170.84 & 99.656 & 52580 \\
$\pi_{4}$ & 126.55 & 28 & 164.84 & 96.15 & 52581 \\
\hline
\end{tabular}

TABLE 4: Numerical result on Example 3.

\begin{tabular}{lcccrc}
\hline Policy & $Q_{s}$ & $m \backslash n$ & $Q \backslash Q^{\prime}$ & $B \backslash B^{\prime}$ & $F \backslash F_{s}$ \\
\hline$\pi_{1}$ & - & 29 & 30 & 169.43 & 98.83 \\
$\pi_{2}$ & - & 27 & 163.67 & 95.47 & 54457 \\
$\pi_{3}$ & 214.89 & 28 & 173.94 & 101.47 & 54458 \\
$\pi_{4}$ & 212.21 & 167.82 & 97.90 & 54368 \\
\hline
\end{tabular}

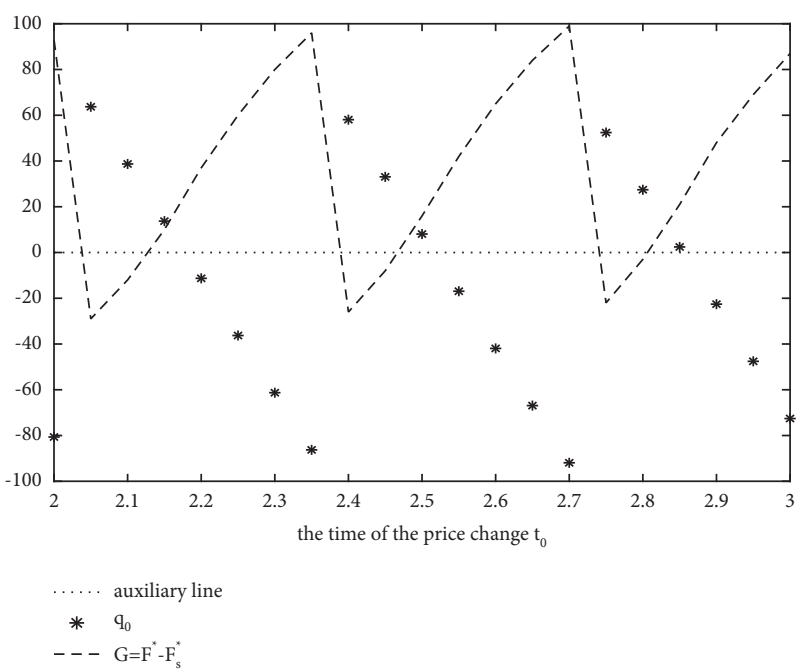

Figure 6: The changes of the $q_{0}$ and $G$ with the price growth rate.

TABLE 5: Impact of parameter $q_{0}$ on the retailer's ordering policy, $c=10.5$.

\begin{tabular}{|c|c|c|c|c|c|c|c|c|}
\hline$t_{0}$ & $q_{0}$ & $\pi_{1}$ & $\pi_{2}$ & $\pi_{3}$ & $\pi_{4}$ & $G^{*}$ & $Q_{s}$ & Optimal policy \\
\hline 2.00 & -80.65 & 56333 & 56334 & 56241 & 56240 & 93 & 216.53 & $\pi_{4}$ \\
\hline 2.05 & 63.71 & $\underline{54490}$ & 54492 & 54520 & $\overline{54519}$ & -29 & - & $\pi_{1}$ \\
\hline 2.10 & 38.71 & $\overline{54480}$ & 54482 & 54492 & 54492 & -12 & - & $\pi_{1}$ \\
\hline 2.15 & 13.71 & 54476 & 54476 & $\underline{54465}$ & $\underline{54465}$ & 10 & 123.60 or 121.06 & $\pi_{3}$ or $\pi_{4}$ \\
\hline 2.20 & -11.29 & 54474 & 54475 & $\overline{54437}$ & $\overline{54438}$ & 36 & 148.21 & $\pi_{3}$ \\
\hline 2.25 & -36.29 & 54470 & 54472 & 54410 & 54412 & 60 & 172.83 & $\pi_{3}$ \\
\hline 2.30 & -61.29 & 54463 & 54464 & $\overline{54384}$ & $\underline{54383}$ & 80 & 197.44 & $\pi_{4}$ \\
\hline 2.35 & -86.29 & 54452 & 54453 & $\underline{54356}$ & $\underline{54356}$ & 96 & 224.73 or 222.06 & $\pi_{3}$ or $\pi_{4}$ \\
\hline 2.40 & 58.06 & $\underline{52609}$ & 52611 & $\overline{52635}$ & $\overline{52635}$ & -26 & - & $\pi_{1}$ \\
\hline 2.45 & 33.06 & $\overline{52600}$ & 52602 & 52608 & 52608 & -8 & - & $\pi_{1}$ \\
\hline 2.50 & 8.06 & $\overline{52596}$ & 52597 & $\underline{52580}$ & 52581 & 16 & 129.18 & $\pi_{3}$ \\
\hline 2.55 & -16.94 & 52595 & 52596 & $\underline{52553}$ & 52554 & 42 & 153.78 & $\pi_{3}$ \\
\hline 2.60 & -41.94 & 52590 & 52592 & $\overline{52527}$ & $\underline{52525}$ & 65 & 178.38 & $\pi_{4}$ \\
\hline 2.65 & -66.94 & 52582 & 52584 & 52500 & 52498 & 84 & 202.98 & $\pi_{4}$ \\
\hline 2.70 & -91.94 & 52570 & 52572 & 52472 & $\underline{52471}$ & 99 & 227.58 & $\pi_{4}$ \\
\hline 2.75 & 52.42 & 50728 & 50730 & 50751 & $\overline{50750}$ & -22 & - & $\pi_{1}$ \\
\hline 2.80 & 27.42 & $\overline{50720}$ & 50722 & 50723 & 50723 & -3 & - & $\pi_{1}$ \\
\hline 2.85 & 2.42 & $\overline{50717}$ & 50719 & $\underline{50696}$ & $\underline{50696}$ & 11 & 134.76 or 132.03 & $\pi_{3}$ or $\pi_{4}$ \\
\hline 2.90 & -22.58 & 50716 & 50717 & $\overline{50668}$ & $\overline{50670}$ & 48 & 159.34 & $\pi_{3}$ \\
\hline 2.95 & -47.58 & 50710 & 50712 & $\overline{50643}$ & $\underline{50641}$ & 69 & 183.93 & $\pi_{4}$ \\
\hline 3.00 & -72.58 & 50701 & 50703 & 50615 & $\overline{50614}$ & 87 & 208.51 & $\pi_{4}$ \\
\hline
\end{tabular}

The underlined values indicate the ordering cost under the optimal ordering strategy. 
TABLE 6: Impact of price increase ratio parameter on the retailer's ordering policy, $t_{0}=2.04$.

\begin{tabular}{|c|c|c|c|c|c|c|c|c|}
\hline$\Delta c / c_{0}(\%)$ & $h$ & $\pi_{1}$ & $\pi_{2}$ & $\pi_{3}$ & $\pi_{4}$ & $G^{*}$ & $Q_{s}$ & Optimal policy \\
\hline 1 & 4.04 & 52506 & 52505 & 52555 & 52554 & -49 & - & $\pi_{2}$ \\
\hline 2 & 4.08 & 53003 & $\underline{53002}$ & 53049 & 53049 & -47 & - & $\pi_{2}$ \\
\hline 3 & 4.12 & 53501 & $\overline{53499}$ & 53543 & 53542 & -43 & - & $\pi_{2}$ \\
\hline 4 & 4.16 & $\underline{53996}$ & $\overline{53998}$ & 54035 & 53034 & -38 & - & $\pi_{1}$ \\
\hline 5 & 4.20 & $\overline{54493}$ & 54494 & 54525 & 54525 & -32 & - & $\pi_{1}$ \\
\hline 6 & 4.24 & $\overline{54990}$ & 54991 & 55015 & 55014 & -24 & - & $\pi_{1}$ \\
\hline 7 & 4.28 & 55487 & 55488 & 55503 & 55502 & -15 & - & $\pi_{1}$ \\
\hline 8 & 4.32 & 55983 & 55984 & 55989 & 55989 & -6 & - & \\
\hline 9 & 4.36 & $\overline{56480}$ & 56482 & 56475 & $\underline{56474}$ & 6 & 117.24 & $\pi_{4}$ \\
\hline 10 & 4.4 & 56977 & 56977 & 56958 & $\overline{56958}$ & 18 & 132.40 or 129.82 & $\pi_{3}$ or $\pi_{4}$ \\
\hline 11 & 4.44 & 57473 & 57474 & $\overline{57441}$ & $\overline{57441}$ & 32 & 144.98 or 142.39 & $\pi_{3}$ or $\pi_{4}$ \\
\hline 12 & 4.48 & 57970 & 57970 & $\overline{57922}$ & $\overline{57922}$ & 48 & 157.55 or 154.96 & $\pi_{3}$ or $\pi_{4}$ \\
\hline 13 & 4.52 & 58466 & 58466 & $\overline{58402}$ & $\overline{58402}$ & 64 & 170.12 or 167.53 & $\pi_{3}$ or $\pi_{4}$ \\
\hline 14 & 4.56 & 58963 & 58963 & $\underline{58881}$ & 58881 & 82 & 182.69 or 180.09 & $\pi_{3}$ or $\pi_{4}$ \\
\hline 15 & 4.60 & 59459 & 59459 & $\overline{59358}$ & $\underline{59358}$ & 101 & 195.25 or 192.65 & $\pi_{3}$ or $\pi_{4}$ \\
\hline 16 & 4.64 & 59995 & 59995 & $\overline{59834}$ & $\overline{59834}$ & 121 & 207.81 or 205.21 & $\pi_{3}$ or $\pi_{4}$ \\
\hline 17 & 4.68 & 60451 & 60451 & $\overline{60308}$ & $\overline{60309}$ & 143 & 220.36 & $\pi_{3}$ \\
\hline 18 & 4.72 & 60948 & 60947 & $\overline{60782}$ & $\underline{60782}$ & 165 & 232.91 or 230.30 & $\pi_{3}$ or $\pi_{4}$ \\
\hline 19 & 4.76 & 61444 & 61443 & $\overline{61254}$ & $\overline{61254}$ & 189 & 245.452 or 242.85 & $\pi_{3}$ or $\pi_{4}$ \\
\hline 20 & 4.80 & 61940 & 61939 & $\overline{61724}$ & $\overline{61725}$ & 215 & 258.99 & $\pi_{3}$ \\
\hline
\end{tabular}

The underlined values indicate the ordering cost under the optimal ordering strategy.

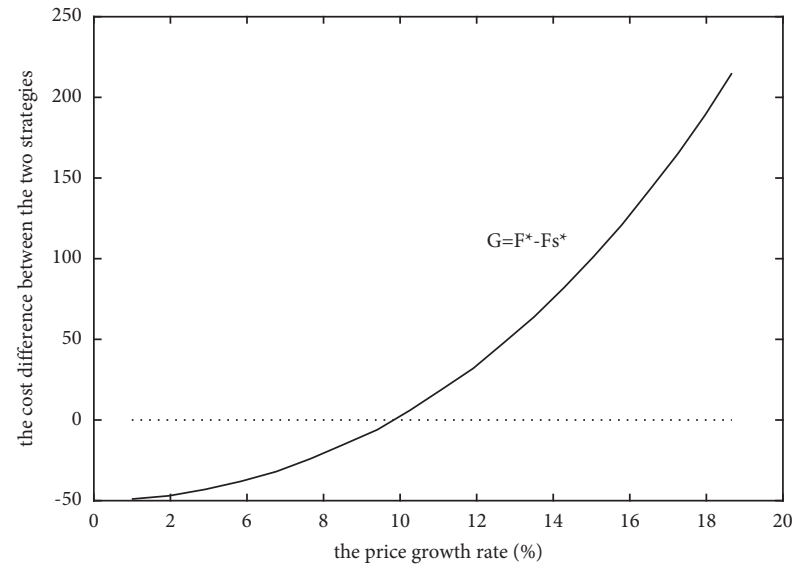

Figure 7: The cost difference between the two strategies with the price growth rate.

Example 3. For the inventory system considered in Example 1 , set $t_{0}=2.33$ and other parameters remain unchanged.

For this example, the remaining stock at $t_{0}$ is $q_{0}=-76.29$.

Similar to Example 1, since $\alpha=38.18>\gamma=0.5916$, and $\beta=-555.16<0$, if a special order is placed, the candidate optimal policy set is $\left\{\pi_{3}, \pi_{4}\right\}$. The numerical results are listed in Table 4, from which we can see that the optimal ordering policy is $\pi_{4}$, and the buyer's inventory cost saving of placing a special order compared to not placing special order is 91 .

In the numerical experiments, we see that the remaining stock $q_{0}$ at $t_{0}$ significantly affects the buyer's ordering policy. Using the same data as those in Example 1, we study the sensitivity analysis.

6.2. Sensitivity Analysis. To better understand the effect of $q_{0}$ on the ordering policy, we conduct a sensitivity analysis of the models by varying the parameter $q_{0}$ while keeping the other parameters fixed as same as in Example 1. The numerical results are presented in Table 5 and Figure 6.

Figure 6 exhibits that $G \geq 0$ when $q_{0} \leq 24$, the buyer should place a special order at $t_{0}$ for this scenario, and the optimal special order quantity decreases with the increase of $q_{0}$. However, when $q_{0}>24$, the buyer makes a special order more cost-saving than not making a special order, and his optimal replenishment policy is one of the candidate optimal policy sets $\pi_{1}, \pi_{2}$.

In general, whether the buyer places a special order not only relates to $q_{0}$, but also relates to the extent of price increase. In order to discuss this issue, we conduct a sensitivity analysis of the model by varying the parameters $c$ and $h$ while keeping the other parameters fixed as same as in Example 1. The numerical results are presented in Table 6 and Figure 7.

From Table 6 and Figure 7, it is not difficult to see that when $q_{0}$ is fixed, a special order is not required when the price increase rate is small. When the price increases to a certain level, placing a special replenishment is the buyer's optimal replenishment strategy. The buyer placing a special replenishment can save costs when the price increase rate is more than $8.5 \%$.

\section{Conclusion and Extensions}

This study investigated the finite horizon inventory system with backordering and a known price increase, which is observed in common items like grain crops, wheat, corn, and soybeans, etc. The supplier allows the buyer to place a special order when the price increases. The objective of this study is to determine whether or not to purchase additional stock and optimal number of orders so that the inventory cost is minimum. For this mechanism, based on the retailers cost analysis, the 
problem is formulated as an optimization model and is solved by optimal techniques. A global optimal solution for the model was obtained through the algorithm given in the paper. From numerical studies, we show that the results of the numerical analysis are consistent with managerial implications and economical common sense. It has been shown that if those basic parameters of the models are given, we can get an optimal ordering policy, and under those basic parameters such as in Example 1 being fixed, the optimal order policy changes with the change of parameters $q_{0}$ and $\Delta c$, and the special replenishment size increases as $q_{0}$ decreases and $c$ increases.

These results in the paper are important to the real world. The prices of goods, for example, wheat or soybeans, will increase after two or three months after harvest. Also when and how much the price increases are generally due to the interval between two normal orders. When buyers have known the price changes, they need to adjust their ordering strategy. Our model provides the decision-maker useful for this mechanism, and the algorithm can provide an optimal replenishment and stocking strategy to the buyers quickly. Numerical experiments were also carried out to illustrate the validity of the models. Sensitivity analysis has been performed in order to determine the robustness of the models presented above.

To make the concerned model more practical, we may introduce the shortages and partial backordering into the model. Further, the time of price change may be stochastic. This will be considered in the future research.

\section{Appendix}

\section{A. Proof of Theorem 1}

The proof of Theorem 1 is divided into two steps.

First, we discuss the optimal solution of problem (3) when $k$ is fixed. Since the constraints are linear, any optimal solution is a KKT point which satisfies one of the following systems. See $[11,25]$.

$$
\left\{\begin{array}{l}
\frac{\partial f}{\partial Q_{1}}=\frac{h}{\lambda}\left(Q_{1}+q\right)-\mu=0, \\
\frac{\partial f}{\partial Q_{i}}=\frac{h}{\lambda}\left(Q_{i}-B_{i-1}\right)-\mu=0, i=2, \ldots, k, \\
\frac{\partial f}{\partial B_{i}}=-\frac{h}{\lambda}\left(Q_{i+1}-B_{i}\right)+\frac{w}{\lambda} B_{i}=0, i=1, \ldots, k-1,
\end{array}\right.
$$

where

$$
f=F\left(k, Q_{1}, \ldots, Q_{k}, B_{1}, \ldots, B_{k-1}\right)-\mu\left(\sum_{i=1}^{k} Q_{i}-\lambda T+q\right)
$$

is the Lagrange function, and $\mu \geq 0$ is the Lagrange multiplier corresponding to the equality constraint.

A straightforward computing of the system of equations gives that

$$
\left\{\begin{array}{l}
Q_{1}=\frac{w \lambda T}{k w+(k-1) h}-q, \\
Q_{2}=\cdots=Q_{k}=\frac{(w+h) \lambda T}{k w+(k-1) h} \triangleq Q, \\
B_{1}=\cdots=B_{k-1}=\frac{h \lambda T}{k w+(k-1) h} \triangleq B,
\end{array}\right.
$$

and thus, $Q_{1}=Q-B-q$.

Substitute the above solutions into formula (2); we obtain the inventory cost over the planning horizon of length $T$ is

$$
\begin{aligned}
F & =c(\lambda T-q)+k A+\frac{k h}{2 \lambda}(Q-B)^{2}+\frac{(k-1) h}{2 \lambda} B^{2} \\
& =c(\lambda T-q)+k A+\frac{w h \lambda T^{2}}{2[k w+(k-1) h]} .
\end{aligned}
$$

Now, we consider the optimal ordering times $k$.

Since

$$
F(k)=c(\lambda T-q)+k A+\frac{w h \lambda T^{2}}{2[k w+(k-1) h]},
$$

by

$$
F^{\prime \prime}(k)=-\frac{w h \lambda T^{2}(w+h)^{2}}{[k w+(k-1) h]}<0,
$$

we know that $F(k)$ is concave in $k$, and its minimum value is reached at the stationary point of the function $F(k)$ which gives

$$
k=T \sqrt{\frac{w \lambda h}{2 A(w+h)}}+\frac{h}{w+h}
$$

Considering $k$ is an integer, we conclude that the optimal ordering time is $k_{1}=\lfloor k\rfloor$ or $k_{2}=\lceil k\rceil$. In detail, if $F\left(k_{1}\right) \leq F\left(k_{2}\right)$, then optimal ordering time is $k_{1}$; otherwise, it is $k_{2}$.

\section{B. Proof of Theorem 2}

Similarly to the proof of Theorem 1 , under the condition that $m$ is fixed, we have

$$
\begin{aligned}
& Q_{1}^{\prime}=\cdots=Q_{m}^{\prime} \triangleq Q^{\prime} \\
& B_{s}=B_{1}^{\prime}=\cdots=B_{m-1}^{\prime} \triangleq B^{\prime} \\
& Q_{s}=\lambda T_{0}-q_{0}-\frac{m \lambda(w+h)\left(h_{0} T_{0}+c_{0}-c\right)}{m h_{0}(w+h)+w h} \\
& Q^{\prime}=\frac{\lambda(w+h)\left(h_{0} T_{0}+c_{0}-c\right)}{m h_{0}(w+h)+w h}, \quad B^{\prime}=\frac{\lambda h\left(h_{0} T_{0}+c_{0}-c\right)}{m h_{0}(w+h)+w h}
\end{aligned}
$$


Considering the requirements that $Q_{s}, Q^{\prime}, B^{\prime} \geq 0$, we conclude that $Q_{s}, Q^{\prime}, B^{\prime}$ given in (54)-(55) is a solution to model (21) provided that $h_{0} T_{0}+c_{0}-c \geq 0$ and

$\left(\lambda T_{0}-q_{0}\right)\left[m h_{0}(w+h)+w h\right]>m \lambda(w+h)\left(h_{0} T_{0}+c_{0}-c\right)$,

i.e., $m(w+h)\left[q_{0} h_{0}+\lambda\left(c_{0}-c\right)\right]<\left(\lambda T_{0}-q_{0}\right) w h$. Obviously, if $q_{0} h_{0}+\lambda\left(c_{0}-c\right) \leq 0$, then (56) holds; otherwise,

$$
m<\frac{\left(\lambda T_{0}-q_{0}\right) w h}{(w+h)\left[q_{0} h_{0}+\lambda\left(c_{0}-c\right)\right]} .
$$

For simplicity, we denote $\alpha=h_{0} T_{0}+c_{0}-c, \beta=q_{0} h_{0}+$ $\lambda\left(c_{0}-c\right), \delta=\left(\lambda T_{0}-q_{0}\right) w h /(w+h)\left[q_{0} h_{0}+\lambda\left(c_{0}-c\right)\right]$. Then $Q_{s}, Q^{\prime}, B^{\prime}$ given by (54), (55) is a solution to problem (21) provided that

$$
\begin{gathered}
\alpha \geq 0 \text { and } \beta \leq 0, \\
\text { or } \alpha \geq 0, \beta>0 \text { and } m<\delta .
\end{gathered}
$$

Otherwise, the solution of problem (21) is

$$
m=0, Q_{s}=\lambda T_{0}-q_{0}, Q^{\prime}=B^{\prime}=0
$$

The desired result follows by combining the discussion above.

\section{Data Availability}

The data used in our numerical experiments are taken randomly, and all used data released in this paper can be used directly.

\section{Conflicts of Interest}

The authors declare that they have no conflicts of interest.

\section{Acknowledgments}

The authors would like to express their sincere gratitude to the academic advisor, Prof. Yiju Wang, for the opportunity, support, and guidance to the research work. He provided his valuable advice during the course of this research.

\section{References}

[1] E. Naddor, Inventory Systems, John Wiley \& Sons, New York, 1966.

[2] R. G. Brown, Decision Rules for Inventory Management, Holt Rinehart \& Winston, New York, 1967.

[3] S. G. Taylor and C. E. Bradley, "Optimal ordering strategies for announced price increases," Operations Research, vol. 33, no. 2, pp. 312-325, 1985.

[4] E. Erel, "The effect of continuous price change in the EOQ," Omega, vol. 20, no. 4, pp. 523-527, 1992.

[5] R. J. Tersine, "Economic replenishment strategies for announced price increases," European Journal of Operational Research, vol. 92, no. 2, pp. 266-280, 1996.

[6] N. H. Shah, "A discrete-time probabilistic inventory model for deteriorating items under a known price increase,"
International Journal of Systems Science, vol. 29, no. 8, pp. 823-827, 1998.

[7] W. Huang, V. G. Kulkarni, and J. M. Swaminathan, "Optimal EOQ for announced price increases in infinite horizon," Operations Research, vol. 51, no. 2, pp. 336-339, 2003.

[8] P. L. Abad, "Quantity restrictions and the reseller's response to a temporary price reduction or an announced price increase," Asia Pacific Journal of Operational Research, vol. 23, no. 01, pp. 1-23, 2006.

[9] C. J. Chung and H. M. Wee, "Retailer's replenishment policy for deteriorating item in response to future cost increase and incentive-dependent sale," Mathematical and Computer Modelling, vol. 57, pp. 535-550, 2013.

[10] L. Y. Ouyang, K. S. Wu, C. T. Yang et al., "Optimal order policy in response to announced price increase for deteriorating items with limited special order quantity," International Journal of Systems Science, vol. 47, no. 1-4, pp. 718-729, 2016.

[11] Y. Wang, X. Sun, and F. Meng, "On the conditional and partial trade credit policy with capital constraints: a Stackelberg model," Applied Mathematical Modelling, vol. 40, no. 1, pp. 1-18, 2016

[12] Y. Wang, H. Gao, and W. Xing, "Optimal replenishment and stocking strategies for inventory mechanism with a dynamically stochastic short-term price discount," Journal of Global Optimization, vol. 70, no. 1, pp. 27-53, 2018.

[13] A. K. Ghosh, "On some inventory models involving shortages under an announced price increase," International Journal of Systems Science, vol. 34, no. 2, pp. 129-137, 2003.

[14] S. Sharma, "On price increases and temporary price reductions with partial backordering," European Journal of Industrial Engineering, vol. 3, no. 1, pp. 70-89, 2009.

[15] D. W. Pentico and M. J. Drake, "A survey of deterministic models for the EOQ and EPQ with partial backordering," European Journal of Operational Research, vol. 214, pp. 1179-1198, 2011.

[16] A. A. Taleizadeh, B. Mohammadi, L. E. Cardenas-Barron, and H. Samimi, "An EOQ model for perishable product with special sale and shortage," International Journal of Production Economics, vol. 145, p. 318-338, 2013.

[17] B. Lev and A. L. Soyster, "An inventory model with finite horizon and price changes," Journal of the Operational Research Society, vol. 30, no. 1, pp. 43-53, 1979.

[18] F. J. Arcelus, T. Pakkala, and G. Srinivasan, "Inventory replenishment for profit maximization over a finite horizon," International Journal of Operations and Quantitative Management, vol. 21, no. 2, pp. 127-139, 2015.

[19] F. J. Arcelus, T. Pakkala, and G. Srinivasan, "Inventory replenishment for profit maximization over a finite horizon under one-time cost changes," Global Business Review, vol. 19, no. 3 , pp. $235-248,2018$

[20] B. Lev and H. J. Weiss, "Inventory models with cost changes," Operations Research, vol. 38, no. 1, pp. 53-63, 1990.

[21] A. A. Taleizadeh and D. W. Pentico, "An economic order quantity model with a known price increase and partial backordering," European Journal of Operational Research, vol. 228, no. 3, pp. 516-525, 2013.

[22] A. A. Taleizadeh, H. R. Zarei, and B. R. Sarker, "An optimal control of inventory under probablistic replenishment intervals and known price increase," European Journal of Operational Research, vol. 257, no. 3, pp. 777-791, 2017.

[23] A. A. Taleizadeh, B. Hazarkhani, and I. Moon, "Joint pricing and inventory decisions with carbon emission considerations, 
partial backordering and planned discounts," Annals of Operations Research, vol. 290, 2020.

[24] C. D. J. Waters, Inventory Control and Management, Wiley, New Jersey, 2003.

[25] Y. Wang, L. Caccetta, and G. Zhou, "Convergence analysis of a block improvement method for polynomial optimization over unit spheres," Numerical Linear Algebra with Applications, vol. 22, no. 6, pp. 1059-1076, 2015. 